\title{
ZTF18aalrxas: A Type IIb Supernova from a Very Extended Low-mass Progenitor
}

\author{
C. Fremling ${ }^{1}\left(\mathbb{1}, \mathrm{H} \mathrm{Ko}^{2}\right.$, A. Dugas ${ }^{1}$, M. Ergon ${ }^{3}$, J. Sollerman ${ }^{3}$ (1), A. Bagdasaryan ${ }^{1}$, C. Barbarino ${ }^{3}$, J. Belicki ${ }^{4}$, E. Bellm ${ }^{5}$ (1),
} N. Blagorodnova ${ }^{6,17}$ (1) K. De ${ }^{1}$, R. Dekany ${ }^{4}$, S. Frederick ${ }^{7}$, A. Gal-Yam ${ }^{8}$ (I) D. A. Goldstein ${ }^{1,18}$ (1), V. Z. Golkhou ${ }^{5,9,19}$,

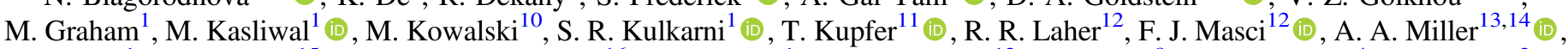

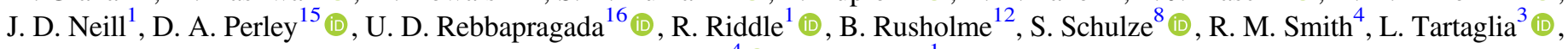
Lin $\mathrm{Yan}^{4}\left(10\right.$, and $\mathrm{Y}$. Yao ${ }^{1}$

${ }^{1}$ Division of Physics, Mathematics and Astronomy, California Institute of Technology, Pasadena, CA 91125, USA; fremling@ caltech.edu

${ }^{2}$ National Central University, Computer Science and Information Engineering, Taoyuan City 32001, Taiwan (R.O.C.)

${ }^{3}$ Department of Astronomy, The Oskar Klein Center, Stockholm University, AlbaNova, SE-10691 Stockholm, Sweden ${ }^{4}$ Caltech Optical Observatories, California Institute of Technology, Pasadena, CA 91125, USA

${ }^{5}$ DIRAC Institute, Department of Astronomy, University of Washington, 3910 15th Avenue NE, Seattle, WA 98195, USA

${ }^{6}$ Department of Astrophysics/IMAPP, Radboud University, Houtlaan 4, 6525 XZ Nijmegen, The Netherlands

Department of Astronomy, University of Maryland, College Park, MD 20742, USA

${ }^{8}$ Benoziyo Center for Astrophysics, The Weizmann Institute of Science, Rehovot 76100, Israel

${ }^{9}$ The eScience Institute, University of Washington, Seattle, WA 98195, USA

${ }^{10}$ Institut für Physik, Humboldt-Universität zu Berlin, Newtonstrasse 15, Berlin D-12489, Germany

${ }^{11}$ Kavli Institute for Theoretical Physics, University of California, Santa Barbara, CA 93106, USA

${ }^{12}$ IPAC, California Institute of Technology, 1200 East California Boulevard, Pasadena, CA 91125, USA

${ }^{13}$ Center for Interdisciplinary Exploration and Research in Astrophysics and Department of Physics and Astronomy, Northwestern University, 2145 Sheridan Road, Evanston, IL 60208, USA

${ }^{14}$ The Adler Planetarium, Chicago, IL 60605, USA

${ }^{15}$ Astrophysics Research Institute, Liverpool John Moores University, Liverpool Science Park, 146 Brownlow Hill, Liverpool L35RF, UK

${ }^{16}$ Jet Propulsion Laboratory, California Institute of Technology, Pasadena, CA 91109, USA

Received 2019 March 21; revised 2019 May 3; accepted 2019 May 6; published 2019 June 5

\begin{abstract}
We investigate ZTF18aalrxas, a double-peaked Type IIb core-collapse supernova (SN) discovered during science validation of the Zwicky Transient Facility. ZTF18aalrxas was discovered while the optical emission was still rising toward the initial cooling peak ( 0.7 mag over 2 days). Our observations consist of multi-band (ultraviolet and optical) light curves (LCs), and optical spectra spanning from $\approx 0.7$ to $\approx 180$ days past the explosion. We use a Monte-Carlo based non-local thermodynamic equilibrium model that simultaneously reproduces both the ${ }^{56} \mathrm{Ni}$-powered bolometric LC and our nebular spectrum. This model is used to constrain the synthesized radioactive nickel mass $\left(0.17 M_{\odot}\right)$ and the total ejecta mass $\left(1.7 M_{\odot}\right)$ of the $\mathrm{SN}$. The cooling emission is modeled using semianalytical extended envelope models to constrain the progenitor radius $\left(790-1050 R_{\odot}\right)$ at the time of explosion. Our nebular spectrum shows signs of interaction with a dense circumstellar medium (CSM), and this spectrum is modeled and analyzed to constrain the amount of ejected oxygen $\left(0.3-0.5 M_{\odot}\right)$ and the total hydrogen mass $\left(\approx 0.15 M_{\odot}\right)$ in the envelope of the progenitor. The oxygen mass of ZTF18aalrxas is consistent with a low $\left(12-13 M_{\odot}\right)$ zero-age main-sequence mass progenitor. The LCs and spectra of ZTF18aalrxas are not consistent with massive single-star SN Type IIb progenitor models. The presence of an extended hydrogen envelope of low mass, the presence of a dense CSM, the derived ejecta mass, and the late-time oxygen emission can all be explained in a binary model scenario.
\end{abstract}

Key words: supernovae: general - supernovae: individual (ZTF18aalrxas)

Supporting material: data behind figure

\section{Introduction}

The light curves (LCs) and spectra of stripped-envelope (SE) supernovae $(\mathrm{SNe})$ can show a wide range of different behaviors. In particular, SNe IIb show intermittent signatures of hydrogen in their photospheric spectra (e.g., Filippenko 1997; Gal-Yam 2017). Furthermore, given early enough observations, the presence of hydrogen tends to be accompanied by an initial cooling phase in the optical LCs before the main radioactively powered peak (e.g., SN 1993J; Filippenko et al. 1993; Nomoto et al. 1993; Schmidt et al. 1993, SN 2011dh; Arcavi et al. 2011; Ergon et al. 2014, 2015 and SN 2013df; Van Dyk et al. 2014; Ben-Ami et al. 2015).

\footnotetext{
17 VENI Fellow.

18 Hubble Fellow.

19 Moore-Sloan, WRF Innovation in Data Science, and DIRAC Fellow.
}

In some $\mathrm{SNe} \mathrm{IIb}$, the cooling signature dominates the early optical LCs during the first weeks following the explosion (SNe 1993J and 2013df). However, in SN 2011dh, there were strong hydrogen features present in early spectra, but the cooling phase lasted less than 5 days (Arcavi et al. 2011). A similar evolution was also seen in, e.g., SN 2008ax (Crockett et al. 2008; Pastorello et al. 2008) and PTF12os (Fremling et al. 2016). The early cooling emission is a result of the SN shock breaking out of the stellar envelope, where the strength and duration of the optical emission is driven by the radius and mass of the envelope material (e.g., Rabinak \& Waxman 2011; Nakar \& Piro 2014; Piro 2015). Thus, an important set of progenitor parameters can be directly probed by studying the early optical emission.

The varying strength of the cooling emission seen in $\mathrm{SNe} \mathrm{IIb}$ can be explained if their progenitors have been stripped of their 
hydrogen envelopes to different degrees. This stripping could either be due to strong stellar winds from very massive stars, with zero-age main-sequence (ZAMS) masses, $M_{\text {ZAMS }}>20 M_{\odot}$ (e.g., Groh et al. 2013), or due to binary interactions (e.g., Yoon et al. 2010, 2017; Claeys et al. 2011), where the stars do not need to be as massive (typically $M_{\text {ZAMS }}<17 M_{\odot}$ ).

A few SNe IIb have been found to have broad and slowly evolving LCs, which are indicative of large ejecta masses that could be consistent with massive stars (see e.g., SN 2003bg; Hamuy et al. 2009; Rubin \& Gal-Yam 2016; and Figure 6 in Fremling et al. 2018). However, for the majority of $\mathrm{SNe} \mathrm{IIb}$ discovered thus far, sample studies tend to favor progenitor mass ranges that are more in line with the expectations for binary models (e.g., Drout et al. 2011; Cano 2013; Taddia et al. 2015, 2018; Lyman et al. 2016; Prentice et al. 2016). Furthermore, for SN 1993J a binary companion has likely been directly observed in post-explosion imaging (Maund \& Smartt 2009 and Fox et al. 2014), providing the most direct evidence for the binary scenario.

Regardless of the origin of $\mathrm{SNe} \mathrm{IIb}$, it is interesting to investigate the main drivers for the differences in the strengths of the cooling signatures and derived progenitor radii (and extended envelope masses) at the time of explosion. Could it be the case that there is a relation between the ZAMS mass of the progenitor and the mass and extent of the envelopes at the time of explosion? In the case of SN 1993J, nebular models indicate a larger ZAMS mass by at least a few solar masses compared to, e.g., SN 2011dh and SN 2008ax (Jerkstrand et al. 2015); also, SN 1993J has one of the strongest cooling signatures observed in any SN IIb. ${ }^{20}$

In this Letter we present a counter-example to this ZAMS mass-driven picture, and instead argue that the structure of the extended envelope is determined by the binary configuration and subsequent evolution. During science validation the Zwicky Transient Facility (ZTF; Bellm et al. 2019; Graham et al. 2019) discovered an SN IIb, ZTF18aalrxas, exceptionally close in time to the explosion. We observed a very strong cooling signature in the optical, almost identical to what was seen in SN 1993J. The cooling phase lasted approximately one week, and was followed by the emergence of a SN IIb LC that qualitatively behaves just like the low-mass SN 2011dh. Latetime spectra also indicate a similarly low ZAMS mass as seen in SN 2011dh $\left(M_{\mathrm{ZAMS}}=12-13 M_{\odot}\right)$, which puts ZTF18aalrxas among the least massive $\mathrm{SNe}$ IIb found to date, while still having a very strong cooling signature.

Our analysis of ZTF18aalrxas spans from $\approx 0.7$ days past explosion until $\approx 180$ days past explosion. In Section 2 we describe our follow-up observations and give details on the data reduction involved. Section 3 presents the multi-band LCs and describes the construction of the bolometric LC of ZTF18aalrxas. A qualitative comparison to other $\mathrm{SNe} \mathrm{IIb}$ shows that the bolometric properties of the $\mathrm{SN}$ are not consistent with the progenitor being very massive (i.e., $\left.M_{\text {ZAMS }}>20 M_{\odot}\right)$. In Section 4 we report our follow-up spectroscopy, confirm the classification of ZTF18aalrxas as an SN IIb, and provide velocity measurements of the SN ejecta. In this section we also use late-time spectroscopy $(\approx 180$ days

\footnotetext{
${ }^{20}$ SN 2013 df also shows a very prominent cooling phase in addition to a very similar spectral evolution to SN 1993J. Morales-Garoffolo et al. (2014) argued that a very low ZAMS mass $\left(12-13 M_{\odot}\right)$ could be consistent with the observed oxygen luminosity at late times, but detailed spectral modeling has not been done.
}

past explosion) to constrain the amount of oxygen and hydrogen in the ejecta. Section 5 presents a Monte-Carlo (MC) based model for the LCs and spectra of ZTF18aalrxas, which we use to constrain the synthesized nickel mass and the total ejecta mass of the explosion, along with the helium core mass of the progenitor. Semi-analytical models are also used in this section to constrain the mass and radius of the extended envelope. Section 6 presents our conclusions, and contains a discussion where we put our observations of ZTF18aalrxas in context with other SNe IIb.

\section{Observations}

\subsection{Detection and Classification}

ZTF18aalrxas was first detected on 2018 April 19.333 $(\mathrm{JD}=2458227.833)$, with the Palomar Oschin Schmidt 48 inch $(\mathrm{P} 48)$ telescope during science validation of the ZTF (Bellm et al. 2019; Graham et al. 2019) and the GROWTH Marshal (Kasliwal et al. 2019). The first detection is in the $g$ band, with a host-subtracted magnitude of $19.59 \pm 0.06 \mathrm{mag}$, at the $\mathrm{J} 2000.0$ coordinates $\alpha=15^{\mathrm{h}} 49^{\mathrm{m}} 11^{\mathrm{s}} .64, \delta=+32^{\circ} 17^{\prime}$ 16 ". 8. Observations $\approx 22 \mathrm{hr}$ earlier on 2018 April 18 give a preexplosion limit of $20.69 \mathrm{mag}$ in $r$ ( $5 \sigma$ limit computed at the expected position of the transient; JD $=2458226.899)$.

Our first spectrum was obtained on 2018 April 25 with the Palomar 60 inch telescope (P60; Cenko et al. 2006) with the Spectral Energy Distribution Machine (SEDM; Blagorodnova et al. 2018). However, this spectrum was largely featureless (with possibly some broad $\mathrm{H} \alpha$ ) and did not lead to a conclusive classification. Follow-up spectra from 2018 April 30 and May 4 showed the emergence of the Balmer series and $\mathrm{He}$ I, characteristic of a SN IIb. Host galaxy emission lines are also present in our spectra $\mathrm{at}^{21} z=0.0582 \pm 0.0003$. Thus, we adopt the distance modulus $\mu=37.10 \mathrm{mag}$, corresponding to a distance ${ }^{22}$ of $263 \mathrm{Mpc}$ to the host galaxy of ZTF18aalrxas.

\subsection{Optical Photometry}

Following the discovery of ZTF18aalrxas, we obtained follow-up photometry during the photospheric phase in $g$ and $r$ with the ZTF Camera on the P48, in gri with the SEDM on the P60, in ugri through the Las Cumbres Observatory Global Telescope Network (Brown et al. 2013), in $g r$ with the Kitt Peak Electron Multiplying CCD on the Kitt Peak 84 inch telescope (Coughlin et al. 2019), and in gri with IO:O on the Liverpool Telescope. The Nordic Optical Telescope (NOT) at La Palma was used to obtain late-time photometry in gri around 150 days past the discovery.

LCs from the P48 come from the ZTF production pipeline (Masci et al. 2019), with limiting magnitudes re-computed using forced point-spread function (PSF) fitting at the expected position of the SN (Y. Yao et al. 2019, in preparation). Stacked P48 LCs were also prepared using SkyPortal (Van der Walt et al. 2019), an online interface to the forced PSF-fit photometry. The stacked LCs were produced by taking the inverse-variance weighted average of the single-epoch PSF photometry in non-overlapping -day windows. LCs from the rest of our optical imaging data have been produced with the

\footnotetext{
21 The quoted redshift is the average that we derive from our 4 Keck I spectra The error is the standard deviation of these estimates.

22 Cosmological parameters from WMAP9 (Hinshaw et al. 2013).
} 


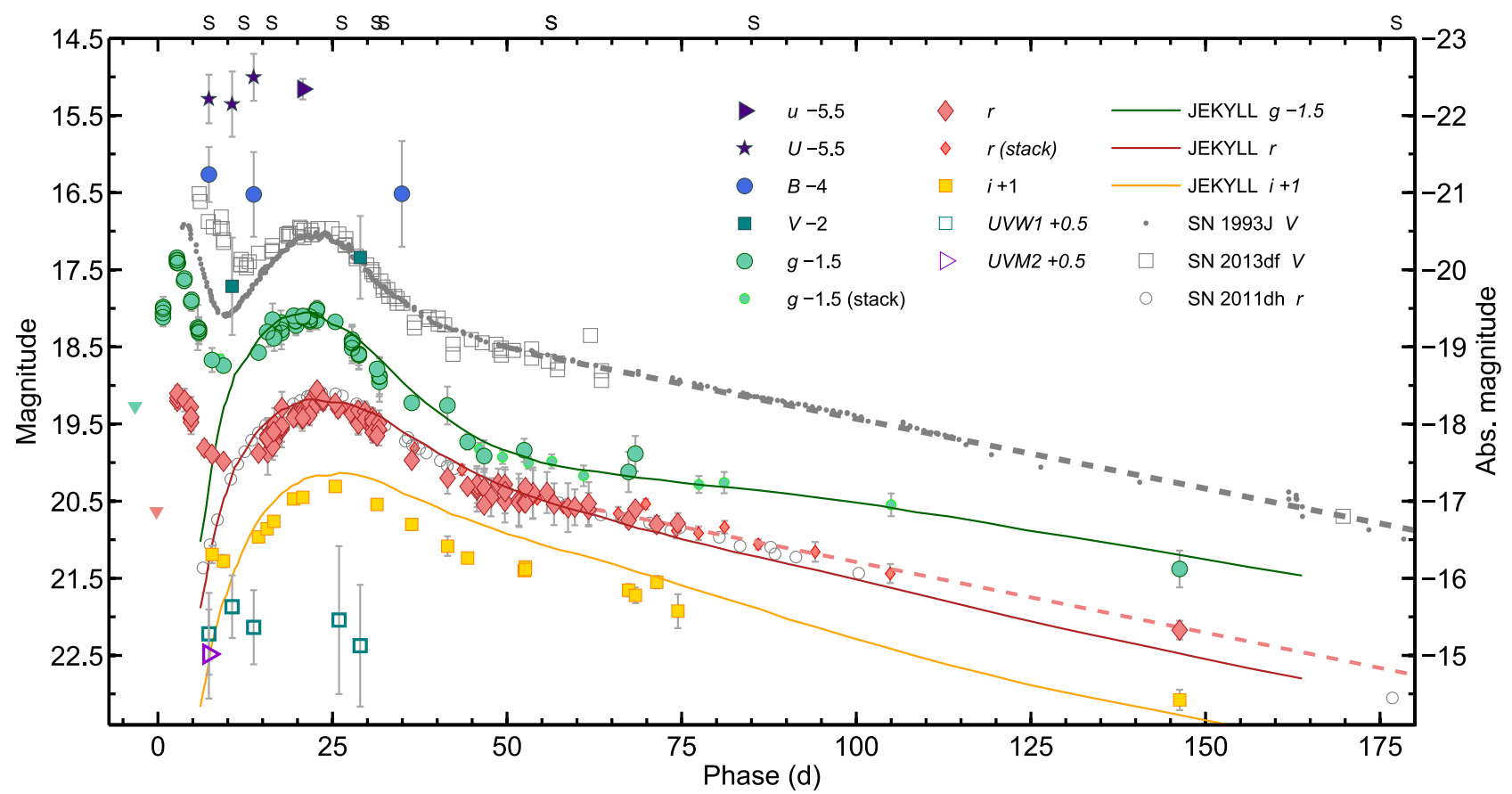

Figure 1. Multi-band LCs of ZTF18aalrxas (colored markers). The $V$-band LCs of SN 2013df (gray empty squares) and SN 1993J (gray dots), and the $r$-band LC of SN 2011dh (gray empty circles) are shown as comparisons. The LC of SN 2011dh has been scaled to the distance and nickel mass of ZTF18aalrxas. The LCs of SN 2013df and SN 1993J have been arbitrarily shifted to match our $V$-band observations of ZTF18aalrxas. The dashed gray line shows a fit to the $V$-band LC of SN 2013df, starting from 50 days past explosion. The dashed red line shows a fit to the $r$-band LC of ZTF18aalrxas, starting from 50 days past explosion. Simulated LCs are shown as solid colored lines (see Section 5). The stacked LC points in the $g$ and $r$-bands contain data from 4 nights of P48 observations for each point. All LCs have been corrected for host-galaxy and MW extinction (see Section 2). The data used to create this figure are available.

image-subtraction pipeline described in Fremling et al. (2016), with template images from the Sloan Digital Sky Survey (SDSS; Ahn et al. 2014). This pipeline produces PSF magnitudes, calibrated against SDSS stars in the field. We have also compared our results to PSF magnitudes calibrated against Pan-STARRS1 (Chambers et al. 2016), finding no significant offsets.

In our analysis we have corrected all photometry for galactic extinction, using the Milky Way (MW) color excess $E(B-V)_{\mathrm{MW}}=0.0192 \mathrm{mag}$ toward the position of ZTF18aalrxas (Schlafly \& Finkbeiner 2011). All reddening corrections are applied using the Cardelli et al. (1989) extinction law with $R_{V}=3.1$. No further host galaxy extinction has been applied, as there is no sign of any $\mathrm{Na}$ ID absorption in any of our spectra. The multi-color LCs of ZTF18aalrxas are shown in Figure 1.

\subsection{Swift-Ultraviolet Optical Telescope Photometry}

A set of ultraviolet (UV) and optical photometry ( $U V W 1$, $U V M 2, U V W 2$, and $U B V$ ) was obtained with the UV Optical Telescope on board Swift (UVOT; Gehrels et al. 2004; Roming et al. 2005). Our first Swift-UVOT observation was performed on 2018 April 25, followed by seven more observations during the photospheric phase of the $\mathrm{SN}$, and two late-time observations (2018 October 24, December 9), in order to estimate the host galaxy contribution.

LCs from Swift-UVOT were produced using HEAs oft (v6.25; HEASARC 2014), as described by Brown et al. (2009), with an aperture radius of $3^{\prime \prime}$. To estimate the host-galaxy contribution at the location of the SN we take a weighted average of the two latest observations. In general the signal-to-noise ratio $(\mathrm{S} / \mathrm{N})$ after host subtraction was very low for this data set. Only data points with $\mathrm{S} / \mathrm{N}>2$ after host subtraction are considered in our analysis.

\subsection{Optical Spectroscopy}

Spectroscopic follow-up of ZTF18aalrxas started on 2018 April 25 ( $\approx 6$ days past discovery) with a spectrum from SEDM mounted on the P60. Further spectra were obtained with the NOT using the Andalucia Faint Object Spectrograph, with the Keck I telescope using the Low Resolution Imaging Spectrograph (LRIS; Oke et al. 1994), with the Dual Imaging Spectrograph on the Apache Point Observatory $3.5 \mathrm{~m}$ telescope, and with the Device Optimized for the Low Resolution on Telescopio Nazionale Galileo. Our latest spectrum was obtained on 2018 October 12 ( $\approx 180$ days past discovery) with LRIS on Keck I.

The LPipe reduction pipeline ${ }^{23}$ (D. A. Perley et al. 2019, in preparation) was used to process the LRIS data. The other spectra were reduced using standard pipelines and procedures for each telescope and instrument. All spectral data and corresponding information will be made available via WISeREP $^{24}$ (Yaron \& Gal-Yam 2012).

\section{Light Curves}

The P48 $g$ - and $r$-band pre-explosion limits along with the UV and optical LCs of ZTF18aalrxas are displayed in Figure 1. Qualitatively, the behavior of ZTF18aalrxas is well matched to

\footnotetext{
23 http://www.astro.caltech.edu/dperley/programs/lpipe.html

24 https://wiserep.weizmann.ac.il/
} 


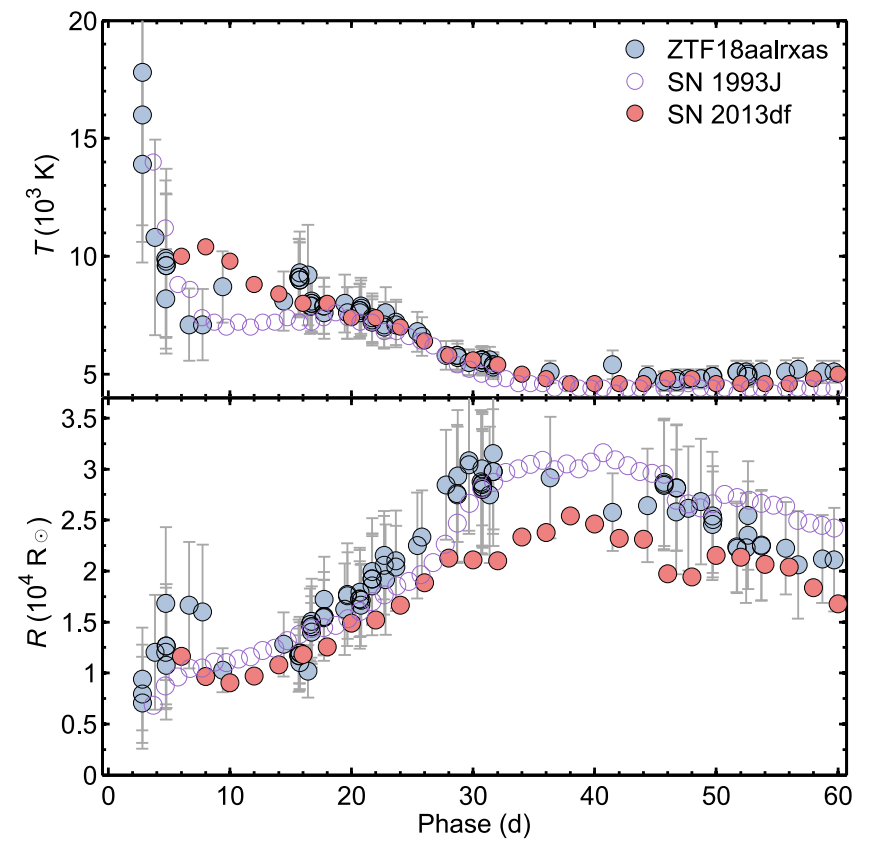

Figure 2. $\mathrm{BB}$ temperature (top panel) and $\mathrm{BB}$ radius (bottom panel) derived from BB fits to the photometry of ZTF18aalrxas (blue circles), SN 1993J (purple unfilled circles), and SN 2013df (red circles).

both SN 1993J and SN 2013df during both the first and second LC peaks. The second peak is also well matched to SN 2011dh, even though SN 2011dh lacked a strong initial cooling phase. For the explosion date of ZTF18aalrxas we have adopted $t_{\text {exp }}=2458227.1 \pm 0.1$ (JD), from our best-fitting model for the early cooling emission ${ }^{25}$ (Section 5.1). The decline rates of the LCs after 50 days past explosion also appear very similar for these objects (dashed lines in Figure 1). We measure the $r$-band decline rate of ZTF18aalrxas to $1.83 \frac{\mathrm{mag}}{100 \text { days }}$ by fitting a firstorder polynomial to the data starting from 50 days past explosion.

A pseudo-bolometric LC for ZTF18aalrxas was constructed by fitting a blackbody (BB) model to the spectral energy distribution (SED) derived from the LCs as a function of time, and integrating the flux of the fitted BB (starting from $2600 \AA$ ). We have weighted our BB fits by the photometric errors. This de-emphasizes the data collected by Swift, which has very large uncertainties. The photometry has been interpolated to the dates of the $r$-band data points, in order to allow the construction of the bolometric LC. Errors have been calculated using a MC method where the BB fits are re-computed for the range of LCs that are allowed by shifting the LC points within the photometric uncertainties (we assume a normal distribution when resampling).

The BB parameters and the pseudo-bolometric LC are shown in Figures 2 and 3, respectively. In Figure 3 we also display the pseudo-bolometric LC of SN 2011dh (data from Ergon et al. 2014) both without scaling and with scaling to match the peak luminosity (nickel mass) of ZTF18aalrxas, as well as scaled pseudo-bolometric LCs of SN 2013df (data from Szalai et al. 2016) and SN 1993J (data from Richmond et al. 1994). The bolometric LCs of SN 2011dh and SN 2013df have been constructed in the same way as the bolometric LC of

\footnotetext{
$\overline{25}$ This value is in good agreement with the result of fitting the early $g$-band flux with a power law.
}

ZTF18aalrxas. When determining the BB parameters and bolometric LC of SN 2013df we have applied a color-based extinction correction with $E(B-V)=0.175 \mathrm{mag}$, based on the observed $g-r$ color 10 days past the $r$-band peak, as described in Taddia et al. (2015). Because the explosion time for SN 2013df is uncertain, we have matched the phase of maximum light of the main peak in the bolometric LC to that of ZTF18aalrxas. ${ }^{26}$ For SN 1993J there are no gri data. Instead we have determined the $\mathrm{BB}$ parameters and constructed the pseudo-bolometric LC via BB fitting of BVRI LCs. As SN 2013df has both gri and BVRI coverage, we have checked that the bolometric LC does not significantly change depending on the filter set in our method. We have adopted the lower value for the extinction $(E(B-V)=0.08 \mathrm{mag})$ used by Richmond et al. (1994) when performing BB fits to the spectral energy distribution (SED) of SN 1993J.

The peak luminosity of ZTF18aalrxas is higher by about a factor of 2.2 compared to $\mathrm{SN} 2011 \mathrm{dh}$, indicating a ${ }^{56} \mathrm{Ni}$ mass $^{27}$ that should be higher by a similar amount, which turns out to be $\approx 0.17 M_{\odot}$. This is very close to the median nickel mass $\left(M^{56} \mathrm{Ni}=0.15 \pm 0.07 M_{\odot}\right)$ of the $\mathrm{SN}$ IIb sample studied by Taddia et al. (2018). The initial cooling phase of ZTF18aalrxas remains very prominent in the bolometric LC, and there is a striking similarity to the cooling phase seen in SN 1993J. The luminosity at the initial peak is also similar to that observed in SN 2013df, but the duration of the cooling phase is shorter in ZTF18aalrxas. After about one week past explosion, the main ${ }^{56} \mathrm{Ni}$-powered LC peak starts to dominate in ZTF18aalrxas, while the cooling dominates for almost one more week in SN 2013df. This indicates a higher mass in the envelope of the progenitor to SN 2013df compared to the progenitor of ZTF18aalrxas (see Section 5.1).

The width of the main bolometric LC peak appears to be very similar for ZTF18aalrxas, SN 1993J, SN 2013df, and SN 2011dh. A bolometric LC well matched to SN 2011dh strongly disfavors a progenitor scenario for ZTF18aalrxas, where the mass loss was dominated by the stellar wind from a very massive star, because the LCs of SN $2011 \mathrm{dh}$ are consistent with stars with $M_{\text {ZAMS }} \leqslant$ $13 M_{\odot}$. Under the assumption that a compact neutron-star remnant is formed, a massive progenitor $\left(M_{\mathrm{ZAMS}} \gtrsim 20 M_{\odot}\right)$ should have a large ejecta mass and slower evolution of the bolometric LC.

From the BB fits to the SED of ZTF18aalrxas we have derived the evolution of $R_{\mathrm{BB}}$, which can be interpreted as a rough approximation of the photospheric radius (bottom panel of Figure 2). $R_{\mathrm{BB}}$ evolves in a very similar way when compared to SN 2013df, and especially when compared to SN 1993J (shown as comparisons). Our earliest measurement is at 2.8 days past explosion, and indicates $R_{\mathrm{BB}}$ in the range $0.8 \pm 0.5 \times 10^{4} R_{\odot}$, which later peaks around $(2.5-3) \times 10^{4} R_{\odot}$ at $\approx 40$ days past explosion. $T_{\mathrm{BB}}$ (top panel of Figure 2) starts out at around $15 \times 10^{3} \mathrm{~K}$ in ZTF18aalrxas at 2.8 days past explosion, but drops quickly to around $7.5 \times 10^{3} \mathrm{~K}$ over the following few days. Again this is remarkably similar to the evolution of SN 1993J, including the rapid decline seen initially. In SN 2013df $T_{\mathrm{BB}}$ stayed around $10^{4} \mathrm{~K}$ for the first 10 days past explosion. Compared to the larger sample of SE SNe presented

\footnotetext{
26 The LC comparison based explosion time estimate for SN 2013df by Van Dyk et al. (2014) would shift the bolometric LC such that the main peak happens a few days later compared to ZTF18aalrxas.

27 Some ${ }^{56} \mathrm{Ni}$-mass estimates for SN $2011 \mathrm{dh}$ are $0.075 \pm 0.025 M_{\odot}$ by Ergon et al. (2014) and $0.07 M_{\odot}$ by Shivvers et al. (2013).
} 


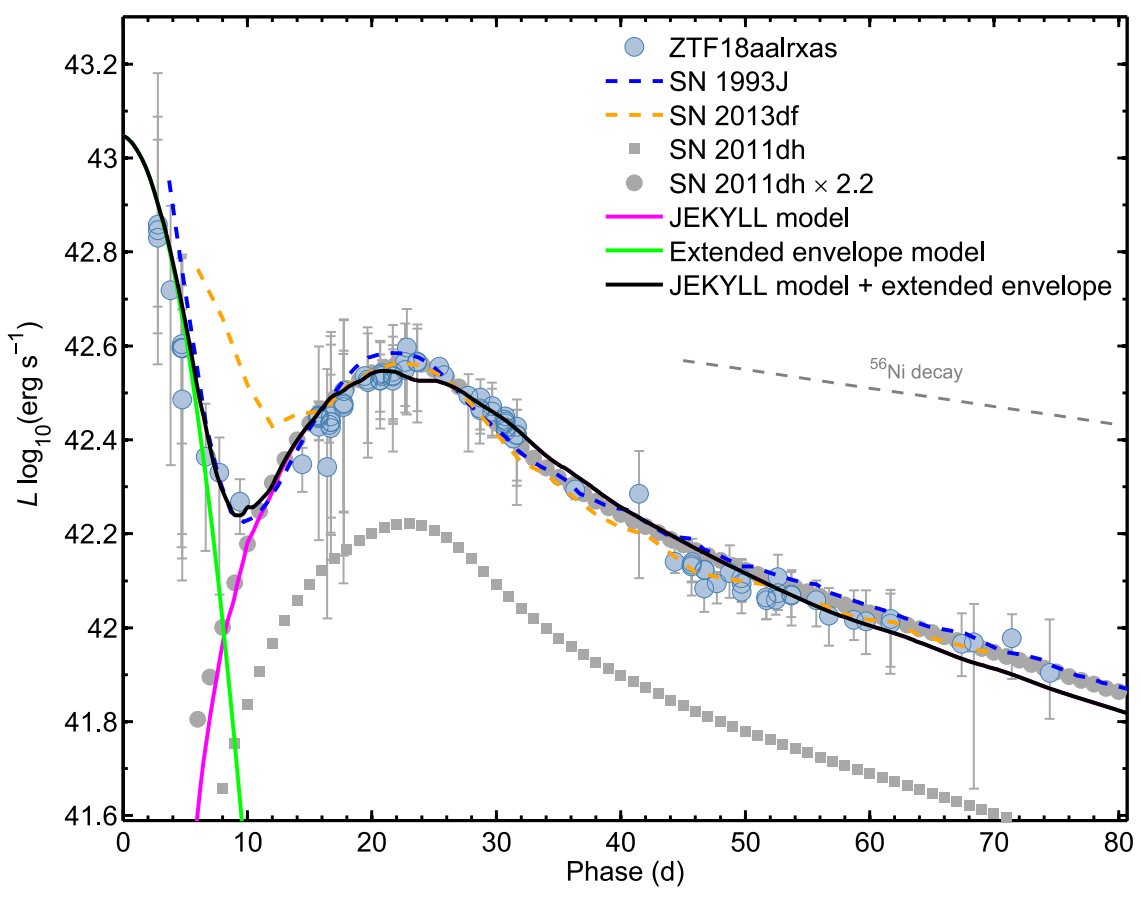

Figure 3. Bolometric LC of ZTF18aalrxas (red circles), compared to the bolometric LCs of SN 2011dh (gray circles and squares), SN 2013df (dashed green line), and SN 1993J (dashed blue line). The LCs of SN 2011dh, SN 2013df, and SN 1993J have been scaled to match the nickel mass of ZTF18aalrxas. The bolometric LC from our non-local thermodynamic equilibrium (NLTE) simulation is shown as a black line. A best-fit semi-analytic model for the cooling emission from an extended envelope (Nagy \& Vinkó, 2016) is shown as a red line (see Section 5). The sum of the NLTE model and the extended envelope model is shown as a pink line.

by Taddia et al. (2018), both $T_{\mathrm{BB}}$ and $R_{\mathrm{BB}}$ of ZTF18aalrxas appear to be very consistent with the averages for SE SNe.

\section{Spectra}

Our spectral sequence (Figure 4) starts out at 7 days past explosion with spectra dominated by the Balmer series. Comparison spectra of SN 2013df, which are very similar to spectra of SN 1993J (see e.g., Morales-Garoffolo et al. 2014; Szalai et al. 2016) are also shown in Figure 4. Our earliest spectrum from SEDM is of low $\mathrm{S} / \mathrm{N}$, but does show a broad feature where $\mathrm{H} \alpha$ is expected, which is also consistent with the NOT spectra taken at 12 and 16 days past explosion, where several of the Balmer lines are present. A spectrum of SN 2013df taken at 12 days past explosion is qualitatively very similar, but slightly bluer, which is consistent with the higher $T_{\mathrm{BB}}$ that we derived in Section 3.

He I signatures appear rather late in ZTF18aalrxas; we observe the simultaneous appearance of He I $\lambda \lambda 5016,5876$, 6678,7065 in absorption at around $7500 \mathrm{~km} \mathrm{~s}^{-1}$ in our spectra past 30 days, with the signatures being quite clear at 56 days past explosion. This evolution is similar to that of SN 2013df and SN 1993J, which also lacked clear He I for the first month. In particular, our LRIS spectrum of ZTF18aalrxas taken at 56 days past explosion is qualitatively very similar to a spectrum taken at a similar epoch of SN 2013df. This clearly solidifies the SN IIb classification of ZTF18aalrxas.

Our spectrum taken at 85 days past explosion shows the emergence of [O I] $\lambda \lambda 5577,6300,6364$ emission lines along with the O I $\lambda 7774$ triplet, hinting that the ejecta are becoming optically thin. The latest spectrum, taken at 177 days past explosion, is clearly in the nebular phase, with prominent [O I] $\lambda \lambda 6300,6364$ seen in emission. There is also broad flat-topped
$\mathrm{H} \alpha$ emission emerging, similar to what was seen in nebular spectra of SN 2013df and SN 1993J. This nebular spectrum is further analyzed and modeled in Section 4.1 in order to constrain the $\mathrm{O}$ and $\mathrm{H}$ envelope masses.

Fe II $\lambda 5169$ is a decent tracer for the photospheric velocity (Dessart \& Hillier 2005). Absorption from this line is present in our spectra taken later than 26 days past explosion. Velocities measured for $\mathrm{Fe}$ II $\lambda 5169, \mathrm{H} \alpha, \mathrm{H} \beta$, and $\mathrm{He} \mathrm{I} \lambda \lambda 5876,7065$ are shown in Figure 5. These measurements were performed by smoothing the spectra and locating the relevant absorption minima. Uncertainties were estimated through a MC simulation where many simulated spectra were created, using smoothed spectra and randomly generated noise reflecting the observed $\mathrm{S} / \mathrm{N}$. The Fe II $\lambda 5169$ velocity of ZTF18aalrxas is in good agreement with the velocity derived from the evolution of the $\mathrm{BB}$ radius (Section 3), which gives $v_{\mathrm{ph}} \approx 8000 \mathrm{~km} \mathrm{~s}^{-1}$ around 26 days past explosion. This velocity is also similar to what can be seen in SN 1993J, SN 2013df, and SN 2011dh at a similar epoch.

\subsection{Nebular Spectroscopy and Oxygen Mass Constraints}

More massive stars produce larger amounts of metals, and the oxygen nucleosynthesis in particular is a strong and monotonic function of $M_{\text {ZAMS }}$ (Woosley \& Heger 2007). This has been exploited by Jerkstrand et al. (2015), who used a grid of simulated spectra to show a strong dependence between the ratio of the [O I] $\lambda \lambda 6300,6364$ line luminosity compared to the total ${ }^{56} \mathrm{Co}$ decay power, for SE SNe in the nebular phase.

We have used the model grid of Jerkstrand et al. (2015) to match our nebular spectrum of ZTF18aalrxas taken at 177 days past explosion, with a focus on the [O I] $\lambda \lambda 6300,6364$ emission (Figure 6). The same model that was used by Jerkstrand et al. (2015) to model the nebular emission of 


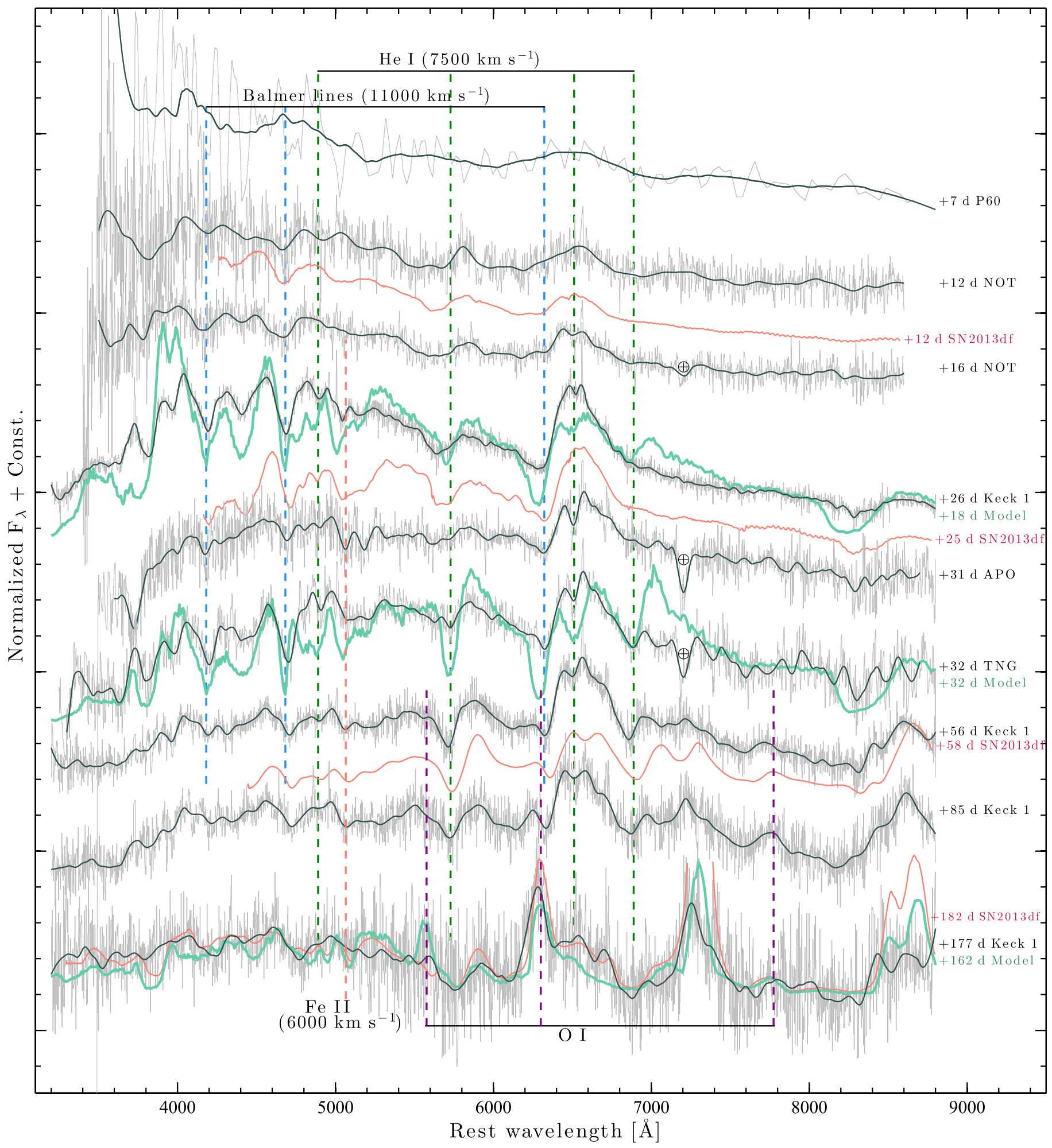

Figure 4. Spectral evolution of ZTF18aalrxas in the wavelength range 3200-8800 A. Black lines show spectra of ZTF18aalrxas smoothed by local polynomial regression. Unsmoothed spectra of ZTF18aalrxas are shown in gray. Comparison spectra of SN 2013df (from Szalai et al. 2016; Shivvers et al. 2019) are shown as red lines. Green lines show simulated spectra (see Section 4). Dashed lines mark the central wavelength of the marked emission lines at rest, or shifted blueward to the velocity specified within parentheses to match the absorption minima associated with the emission lines. The Ca II emission around $7300 \AA$ A has been clipped in the spectrum of SN 2013df. A telluric feature near $7200 \AA$ is present in the spectra taken at $+16,+31$, and +32 days.

(A FITS file for this figure is available.)

SN 2011dh (model 12C in Jerkstrand et al. 2015) is also quite well matched to ZTF18aalrxas. The peak luminosity of the [O I] $\lambda \lambda 6300,6364$ line is slightly higher in our observation, but not as high as what would be expected for a $M_{\text {ZAMS }}=$ $13 M_{\odot}$ model. Thus, we conclude that the $\mathrm{O}$ mass should be in the range $0.3-0.5 M_{\odot}$ (O mass values from Jerkstrand et al. 2015), 


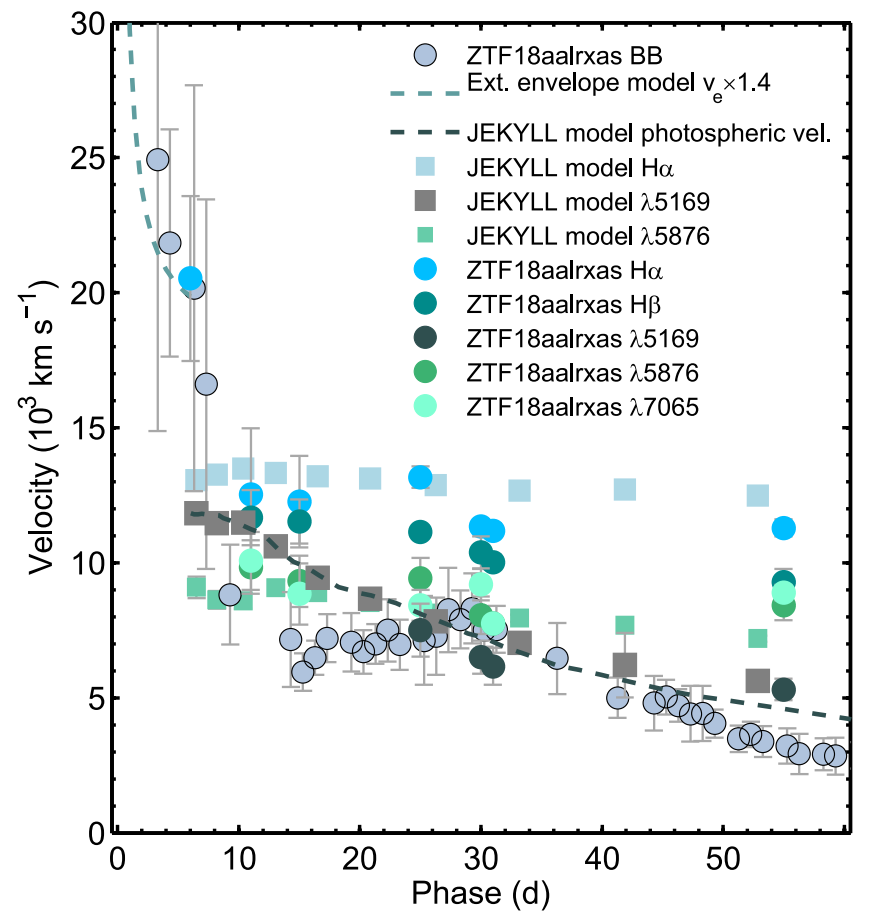

Figure 5. Line velocities derived from absorption minima in the spectra of ZTF18aalrxas (colored circles) and our simulated spectra from JEKYLL (colored squares). The expansion velocity derived from BB fits to the photometry of ZTF18aalrxas is shown as steel blue circles with a black outline. The photospheric velocity of our extended envelope model (based on Nakar \& Piro 2014) is shown as a dashed blue line, and the photospheric velocity in our JEKYLL model is shown as a dashed gray line.

which for these models indicate $M_{\mathrm{ZAMS}}=12-13 M_{\odot}$ for the progenitor of ZTF18aalrxas.

\subsection{Hydrogen Mass Constraints}

Our spectrum of ZTF18aalrxas taken at 177 days past explosion shows a clear broad excess emission, around the region of $\mathrm{H} \alpha$, when compared to our model spectra (Figure 6). Furthermore, the profile of this emission is flat; this is almost identical to what was seen in SN 2013df at a very similar epoch (see Figure 4), and somewhat later in SN 1993J (see e.g., Patat et al. 1995). This feature gradually becomes clearer with time in SN 1993J and SN 2013df, and eventually completely dominates the $\mathrm{H} \alpha$ region after around 600 days past explosion (e.g., Maeda et al. 2015). It is not possible to explain the luminosity and broadness of this line at such late epochs with a ${ }^{56}$ Co-powered nebular model such as in Jerkstrand et al. (2015). The flat and broad profile indicates that the emitting region has a shell-like geometry, and that interaction with circumstellar material (CSM) is the powering mechanism (Chevalier \& Fransson 1994).

If we combine the $12 \mathrm{C}$ model spectrum from Jerkstrand et al. (2015), with a spectrum of SN 1993J (from Matheson et al. $2000 \mathrm{a}, 2000 \mathrm{~b}$ ) taken at 976 days past explosion (where there is very little contribution from ${ }^{56} \mathrm{Co}$-powered metal lines remaining), we find that the excess broad emission around $\mathrm{H} \alpha$ can be almost perfectly reproduced. Furthermore, this combined (12C + SN 1993J) model is at the same time generally better matched to the observed spectrum in the entire blue part of the spectrum $(\lambda<6000 \AA)$. By integrating the broad $\mathrm{H} \alpha$ in the spectrum of SN 1993J in the combined $12 \mathrm{C}+\mathrm{SN} 1993 \mathrm{~J}$ model after it was matched to ZTF18aalrxas at 177 days past explosion, we estimate the $\mathrm{H} \alpha$ luminosity of ZTF18aalrxas to $L_{\mathrm{H} \alpha} \approx 1.22 \times$ $10^{39} \mathrm{erg} \mathrm{s}^{-1}$ at this epoch. ${ }^{28}$

Patat et al. (1995) have constructed a model to estimate the $\mathrm{H}$ mass of SN 1993J based on the $\mathrm{H} \alpha$ luminosity (see their Equation (2)). This equation, applied to their $\mathrm{H} \alpha$ luminosities between 171 and 367 days past explosion, results in virtually the same upper limit for the $\mathrm{H}$ envelope mass for all epochs $\left(M_{\mathrm{H}} \approx 0.2 M_{\odot}\right)$. Because the mass estimate is not increasing over time, this favors a scenario where the emitting region is the unshocked ejecta in the CSM model; the entire hydrogen envelope in the ejecta is contributing to the $\mathrm{H} \alpha$ luminosity (see also Fransson et al. 2005). A detailed discussion on SN 2013df along similar lines can be found in Maeda et al. (2015), who were also able to constrain the composition of the emitting region to $40 \% \mathrm{H}$ and $60 \% \mathrm{He}$ in mass (which gives the electron density $1.4 n_{\mathrm{H}^{+}}$). If this electron density is applied to SN $1993 \mathrm{~J}$, the envelope mass estimate becomes $M_{\mathrm{H}} \approx 0.14 M_{\odot}$. For SN 2013df Maeda et al. (2015) derived $M_{\mathrm{H}} \approx 0.2 M_{\odot}$. If we apply Equation (2) in Patat et al. (1995) to ZTF18aalrxas, assuming the electron density of SN 2013df, and the $\mathrm{H} \alpha$ velocity at the red edge of the line $\left(v_{e}=11,500 \mathrm{~km} \mathrm{~s}^{-1}\right.$, measured from our nebular spectrum), we find $M_{\mathrm{H}} \approx 0.15 M_{\odot}$, which gives the total envelope mass $(\mathrm{H}+\mathrm{He}) M_{\mathrm{env}} \approx 0.38 M_{\odot}$.

In conclusion, the hydrogen envelope masses of SN 1993J and ZTF18aalrxas appear to be very similar, while the hydrogen envelope mass of SN 2013df is somewhat larger. This finding is consistent with SN 2013df having a longer lasting early cooling phase (see Section 5.1). We also note that because the CSM contribution to the $\mathrm{H} \alpha$ line appears to be significant already at $\sim 170$ days past explosion in these $\mathrm{SNe},{ }^{29}$ a dense CSM is required, and they must be exploding when strong mass loss is ongoing; for SN 2013df, Maeda et al. (2015) estimated that during the final $\sim 800 \mathrm{yr}$ before the explosion the mass-loss rate was $\sim 5 \times 10^{-5} M_{\odot} \mathrm{yr}^{-1}$. For SN $1993 \mathrm{~J}$ a mass-loss rate of $\sim 4 \times 10^{-5} M_{\odot} \mathrm{yr}^{-1}$ was derived by Fransson et al. (1996), based on X-ray and radio observations. Given the similarity of ZTF18aalrxas to these objects in terms of the late-time $\mathrm{H} \alpha$ line produced by CSM interaction, a comparable mass-loss rate would be expected.

\section{Modeling and Progenitor Constraints}

\subsection{Modeling the Cooling Emission}

Figure 7 shows the gri-band LCs of ZTF18aalrxas up until $\approx 8$ days past explosion. We detected ZTF18aalrxas while the $g$-band luminosity was still rising toward the initial peak in the LC. This early emission can be modeled as the result of the SN shock breaking out from a progenitor surrounded by an extended envelope (e.g., Rabinak \& Waxman 2011; Nakar \& Piro 2014; Piro 2015). In these models, the SN shock heats the extended material to very high temperatures $\left(T>10^{5} \mathrm{~K}\right)$, after which the material rapidly starts to cool. This means that what looks like an initial rise to a peak in the optical is actually a

\footnotetext{
28 The accuracy of this estimate is motivated by the fact that there is little velocity evolution in the red- and blue-edge velocities of the broad $\mathrm{H} \alpha$ feature between 171 and 1766 days past the explosion in SN 1993J (Patat et al. 1995; Matheson et al. 2000a, 2000b).

${ }^{29}$ Fang \& Maeda (2018) have argued that at $~ 200$ days past explosion, the $\mathrm{H} \alpha$-like structure in most $\mathrm{SNe} \mathrm{IIb}$ is predominately powered by radioactive decay. However, we have here removed the contribution from a radioactively powered SN using the C12 model from Jerkstrand et al. (2015); we consider the excess that cannot be explained by the model as CSM-powered emission.
} 


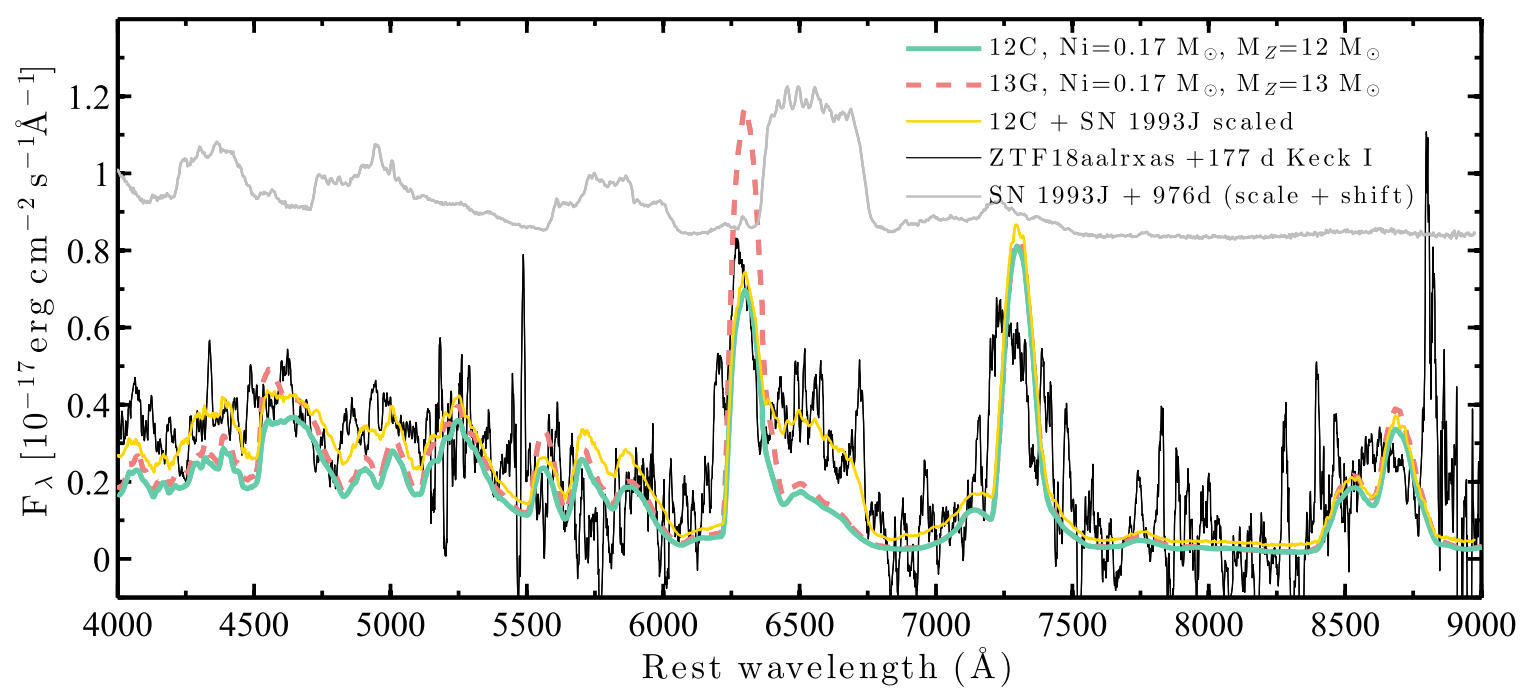

Figure 6. Nebular spectrum of ZTF18aalrxas obtained at +177 days (black line) compared to simulated spectra. One simulation is shown for $M_{\mathrm{ZAMS}}=13 M_{\odot}(13 \mathrm{C}$; red dashed line) and another for $M_{\mathrm{ZAMS}}=12 M_{\odot}\left(12 \mathrm{C}\right.$; green line). The observed spectrum is consistent with $M_{\mathrm{ZAMS}}$ in the range 12-13 $M_{\odot}$. The spectrum of ZTF18aalrxas has been flux calibrated using our $r$-band photometry (extrapolated to 177 days past explosion). All simulations have been scaled to the epochs of the spectra according to the prescriptions of Jerkstrand et al. (2015). A spectrum taken at 976 days past explosion of SN 1993J (from Matheson et al. 2000a, 2000b) is shown as a gray line. This spectrum of SN 1993J scaled to match ZTF18aalrxas around $6500 \AA$, and combined with the 12C model, is shown as a yellow line.

temperature effect. The SED of the SN gradually moves into the optical range as the ejecta cool, resulting in rising LCs, but the total bolometric luminosity is actually declining. Regardless, having data points on the optical rise to the first peak is a highly significant observation, as it allows a very accurate explosion time estimate, which is rare for double-peaked $\mathrm{SNe}$ IIb. For SN 2016gkg, which had a comparable cooling phase duration to SN 2011dh, there is an extremely early discovery (0.1-0.2 days past the explosion; Bersten et al. 2018). However, in both SN 1993J and SN 2013df the first points on the LCs are on the decline from the initial LC peaks.

In order to estimate the properties of the extended material that gives rise to the initial LC peak of ZTF18aalrxas, we have used the Markov Chain MC (MCMC) method in the Python emcee package (Foreman-Mackey et al. 2013), and the model by Nakar \& Piro (2014). This is a one-zone model for the extended envelope, with the mass in the envelope $M_{\text {env }}$ concentrated at a radius $R_{\text {env }}$. For the opacity of the envelope material we assume $\kappa=0.3 \mathrm{~cm}^{2} \mathrm{~g}^{-1}$. The core mass, $M_{c}$, the time of explosion $t_{\exp }$, and the velocity of the extended material, $v_{e}$, also enter as parameters. For $M_{c}$ we use the He core mass from our model of the main bolometric LC peak (Section 5.2). For the explosion energy we use $E=10^{51} \mathrm{erg}$, which gives $v_{e}$ that is reasonable ${ }^{30}$ compared to the velocities derived from the evolution of $R_{\mathrm{BB}}$ and the $\mathrm{H} \alpha$ velocities in our earliest spectra (Figure 5). The result of the MCMC simulation is $\quad M_{\text {env }}=4.3_{-0.13}^{+0.14} \times 10^{-2} M_{\odot}, \quad R_{\text {env }}=0.73_{-0.02}^{+0.03} \times 10^{14} \mathrm{~cm}$ $\left[1050_{-30}^{+40} R_{\odot}\right]$, and $t_{\exp }=2458227.1_{-0.1}^{+0.1}$ (JD). Uncertainties represent $68 \%$ confidence intervals. The range of models allowed are visualized in Figure 7. These models indicate that the contribution from the cooling envelope to the LCs drops rapidly after 6 days past explosion.

We have also used the model by Nagy \& Vinkó (2016) to model the early bolometric LC (see Figure 3 ). This model is fit by eye, and we are able to achieve an excellent match to the

\footnotetext{
${ }^{30}$ For an envelope in hydrostatic equilibrium the velocity of the extended material should be lower by a factor $1.4 \pm 0.1$ compared to the photospheric velocity (Nakar \& Sari 2010; Nakar \& Piro 2014).
}

early declining LC for, $M_{\mathrm{env}}=5.4 \times 10^{-2} M_{\odot}, R_{\mathrm{env}}=0.55 \times$ $10^{14} \mathrm{~cm}\left[\approx 790 R_{\odot}\right]$, with an initial kinetic energy $E_{k}=0.1 \times$ $10^{51} \mathrm{erg}$, and initial thermal energy $E_{\mathrm{th}}=0.05 \times 10^{51} \mathrm{erg}$, and $\kappa=0.3 \mathrm{~cm}^{2} \mathrm{~g}^{-1}$. While these values give the nicest looking fit for the bolometric LC, models that are closer to the result from the MCMC fit based on the Nakar \& Piro (2014) model are still generally within the error bars on our early bolometric data points. The expansion velocity of the outer ejecta in the bestfitting Nagy \& Vinkó (2016) model is $\approx 20,000 \mathrm{~km} \mathrm{~s}^{-1}$, which is consistent with the early BB evolution and the $\mathrm{H} \alpha$ velocity in our earliest spectrum (Figure 5). Thus, we conclude that both of these models are generally in agreement. The early emission of ZTF18aalrxas can be very well reproduced as the cooling emission from a low mass and very extended envelope.

Figure 3 shows that the duration of the first LC peak is markedly shorter in ZTF18aalrxas ( $\approx 6$ days) compared to SN 2013df ( $\approx 12$ days). A significant difference in cooling phase duration, in the context of these semi-analytical models, can most easily be explained by a difference in the envelope mass around the progenitor at the time of explosion. Using the Nagy \& Vinkó (2016) model, we find that $M_{\text {env }}=11 \times$ $10^{-2} M_{\odot}$ and $R_{\text {env }} \approx 370 R_{\odot}$ is required to model the early (unscaled) bolometric emission of SN $2013 \mathrm{df}^{31}{ }^{31} \mathrm{We}$ are using a higher value for $E(B-V)$, but our values are still comparable to the values $\left(M_{\mathrm{env}}=8.0 \times 10^{-2} M_{\odot}, R_{\mathrm{env}} \approx 160 R_{\odot}\right)$ derived using the same model for SN 2013df by Szalai et al. (2016). By similar reasoning it can be argued that the extended envelope mass and radius of SN 1993J should be very similar to that of ZTF18aalrxas; qualitatively the early LCs behave very similarly, with the turnover from cooling to ${ }^{56} \mathrm{Ni}$ power happening at a very similar epoch for both SNe. By fitting the unscaled bolometric LC of SN 1993J, we derive $M_{\text {env }} \approx 6 \times$ $10^{-2} M_{\odot}, R_{\mathrm{env}} \approx 500 R_{\odot}$.

In conclusion, the hydrogen envelopes of ZTF18aalrxas and SN 1993J appear to be very similar, while the envelope of

\footnotetext{
${ }^{31}$ We use $E_{k}=0.3 \times 10^{51} \mathrm{erg}$, and $E_{\mathrm{th}}=0.145 \times 10^{51} \mathrm{erg}$. These values result in an expansion velocity of $\approx 20,000 \mathrm{~km} \mathrm{~s}^{-1}$ in the extended shell, which is roughly consistent with the $\mathrm{H} \alpha$ velocity in early spectra of SN $2013 \mathrm{df}$.
} 


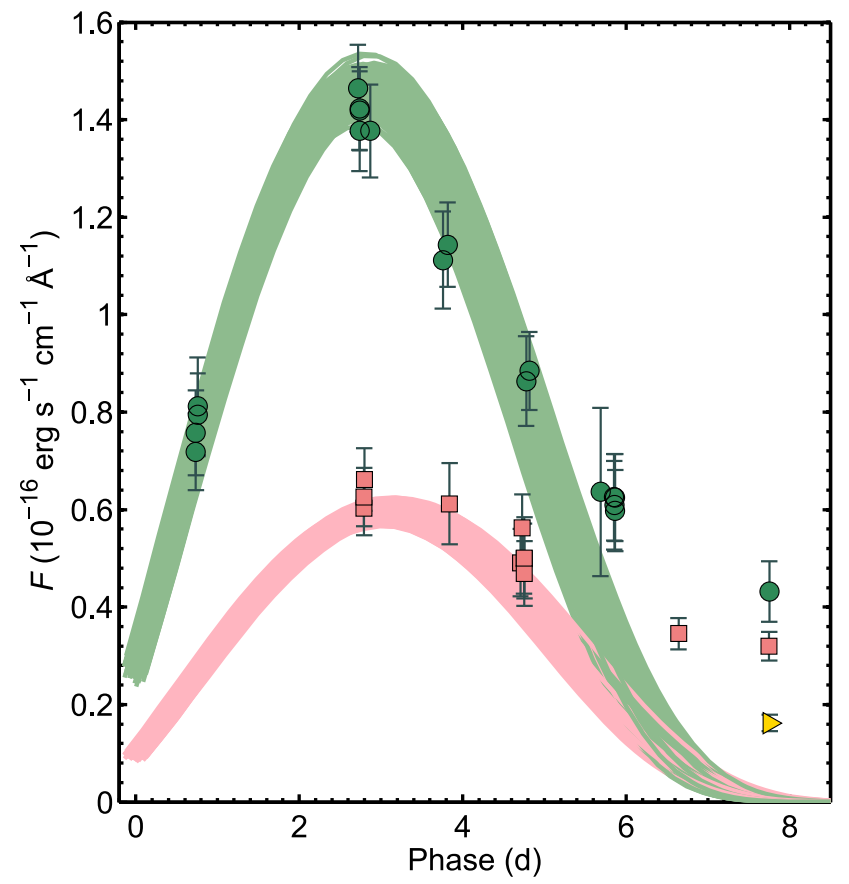

Figure 7. Cooling emission model fit to the early gri LCs of ZTF18aalrxas (colored markers). The range of models from our MCMC simulation based on the model by Nakar \& Piro (2014) are shown as solid lines. The range of the models span the $68 \%$ confidence intervals in the MCMC simulation.

SN 2013df is slightly more massive. In Section 4.1 we arrived at the same qualitative conclusion, based on our nebular spectroscopy. However, the hydrogen masses were somewhat larger. This is likely a calibration issue of the semi-analytical LC models; we consider the nebular constraints as more indicative of the total $\mathrm{H}$ mass at the time of explosion, because these estimates are significantly less model dependent (e.g., they do not depend on assumptions about kinetic or explosion energies).

\subsection{Modeling LCs and Spectra}

As the LC contribution from the cooling of the extended envelope of ZTF18aalrxas becomes insignificant at 6 days past explosion (still almost 20 days before the main bolometric LC peak), the ${ }^{56} \mathrm{Ni}$-powered part of the bolometric LC can be modeled independently in order to constrain the explosion energy $(E)$, the mass of the He core $\left(M_{\mathrm{He}}\right)$, and the ${ }^{56} \mathrm{Ni}$ mass $\left(M_{\mathrm{Ni}}\right)$.

For this purpose we have used the same model (12C) that we found to agree well with our nebular spectrum of ZTF18aalrxas in Section 4.1. We have calculated the evolution of the $12 \mathrm{C}$ model between 1 and 200 days $^{32}$ with the MC based non-local thermodynamic equilibrium (NLTE) code JEKYLL (Ergon et al. 2018). More precisely, this means that we have taken model 12C from Jerkstrand et al. (2015), rescaled it homologously to 1 day, and then evolved it with JEKYLL. This JEKYLL version of the $12 \mathrm{C}$ model shows a good agreement with SN 2011dh throughout the evolution, and will be discussed in more detail in M. Ergon et al. (2019, in preparation). Here we compare it to ZTF18aalrxas, and find that it reproduces the main peak in the bolometric LC of

\footnotetext{
32 In this Letter our model comparisons start from 6 days past explosion, as this is where ${ }^{56} \mathrm{Ni}$ starts to dominate over cooling.
}

ZTF18aalrxas extremely well, after it is scaled by a factor of 2.2. Because the nickel mass of the $12 \mathrm{C}$ model is $M_{\mathrm{Ni}}=$ $0.075 M_{\odot}$, we can estimate the nickel mass for ZTF18aalrxas to $M_{\mathrm{Ni}}=0.17 M_{\odot}$. Furthermore, when combined with the Nagy \& Vinkó (2016) model for the early cooling emission, the full bolometric LC can be reproduced (Figure 3). The rest of the parameters for the $12 \mathrm{C}$ model are $M_{\mathrm{He}}=3.1 M_{\odot}$ and $E=$ $0.68 \times 10^{51} \mathrm{erg}$. The photospheric velocity of the $12 \mathrm{C}$ JEKYLL model is also well matched to the velocities derived from Fe II $\lambda 5169$ in ZTF18aalrxas (Figure 5).

The gri LCs for the 12C JEKYLL model are compared to those of ZTF18aalrxas in Figure 3. The $g$ - and $r$-bands are well matched, in particular with the flattening between 50 and 100 days past explosion observed in the $g$-band for ZTF18aalrxas (also seen in the model $g$-band LC). The $i$-band stays somewhat too bright for a few months following maximum light in the model, but becomes consistent with our observations again when the ejecta are in the nebular phase $(\approx 150$ days past explosion). In general, the broadband optical emission from the 12C JEKYLL model appears well matched to ZTF18aalrxas.

A set of simulated spectra ${ }^{33}$ based on the 12C JEKYLL model are shown in Figure 4. While the observed and modeled spectra are qualitatively similar, with comparable absorption line velocity evolution (Figure 5), we do note some interesting discrepancies. Most importantly, clear He lines appear earlier in the 12C JEKYLL model. Our spectrum of ZTF18aalrxas taken at 26 days past maximum still has very weak He signatures, while the model has already developed clear He I $\lambda 7065$ emission at 18 days past explosion. In later spectra, the He I lines continue increasing in strength, and at 32 days past explosion they are clearly much stronger in the 12C JEKYLL model compared to what we see in ZTF18aalrxas. The absorption from $\mathrm{H} \alpha$ is also stronger in the 12C JEKYLL model compared to our observations of ZTF18aalrxas. However, $\mathrm{H} \alpha$ emission is stronger in ZTF18aalrxas.

These observational facts can likely be explained if the hydrogen envelope of ZTF18aalrxas is more massive compared to that of the $12 \mathrm{C}$ model, such that the He emission is blocked for a longer time early on. ${ }^{34}$ The $\mathrm{H} \alpha$ velocities in the $12 \mathrm{C}$ JEKYLL model are also somewhat faster, indicative of a lower $\mathrm{H}$ mass (for the same explosion energy). A higher $\mathrm{H}$ mass, especially in the extended envelope of the progenitor, is also supported by the early cooling emission that is much stronger in ZTF18aalrxas compared to SN 2011dh.

As the ejecta become optically thin, and the signatures from $\mathrm{H}$ and He decrease, the 12C JEKYLL model becomes very well matched to ZTF18aalrxas, except for possibly some excess $\mathrm{H} \alpha$ (see the spectrum taken at 177 days past explosion, and the discussion in Section 4.2). This should not be surprising as the same ejecta model is used as in the original $12 \mathrm{C}$ model by Jerkstrand et al. (2015) that we investigated in Section 4.1. The $\mathrm{O}$ mass in the $12 \mathrm{C}$ model is $0.3 M_{\odot}$, and the ZAMS mass is $12 M_{\odot}$.

\footnotetext{
33 In Figure 4, the model spectra have been scaled to the nickel mass of ZTF18aalrxas (factor of 2.2). Furthermore, when the epoch of the model spectrum differs from the observed spectrum of ZTF18aalrxas used in the comparison, the model spectrum has been scaled using the model $r$-band LC, so that synthetic photometry on the model spectrum matches the model $r$-band LC at the epoch of the ZTF18aalrxas spectrum.

${ }^{34}$ Some adjustment of the ${ }^{56} \mathrm{Ni}$ mixing throughout the ejecta could also result in a more delayed appearance of the He lines.
} 
In conclusion, the $12 \mathrm{C}$ model, while it could use some fine tuning of the structure and mass of the $\mathrm{H}$ envelope, can be used to robustly constrain the ${ }^{56} \mathrm{Ni}$ mass and $\mathrm{He}$ core mass of ZTF18aalrxas.

\section{Discussion and Conclusions}

We have performed a thorough analysis of the optical emission from ZTF18aalrxas, finding that the progenitor of this SN had, in the context of SE SNe (e.g., Taddia et al. 2018), a low mass $\left(M_{\text {ZAMS }}=12-13 M_{\odot}\right)$ and was surrounded by a very extended envelope $\left(R_{\text {env }}=790-1050 R_{\odot}\right)$, with a mass $\left(M_{\mathrm{env}}=0.04-0.15 M_{\odot}\right)$ at the time of explosion.

Our ZAMS mass results are based on the $12 \mathrm{C}$ model, originally presented by Jerkstrand et al. (2015), and here extended in time with a new MC NLTE code (JEKYLL; Ergon et al. 2018). The $12 \mathrm{C}$ JEKYLL model is able to reproduce the main bolometric LC of ZTF18aalrxas very well, while at the same time also reproducing the [O I] $\lambda \lambda 6300,6364$ emission in our nebular spectrum of the SN. These two constraints are usually obtained from separate modeling codes: one that simulates the bolometric LC, and one that simulates the nebular phase spectra. In general, these simulations do not use the same progenitor model. Our new 12C JEKYLL model offers strong evidence for a very low ZAMS-mass progenitor for ZTF18aalrxas in the $12-13 M_{\odot}$ range, which is consistent with the ZAMS mass predicted for SE SNe by binary evolution modeling (e.g., Yoon et al. 2017), and not consistent with SE SNe produced through wind-driven mass loss from massive stars (e.g., Groh et al. 2013). Thus, it is very likely that the progenitor of ZTF18aalrxas was part of a binary system.

Furthermore, our nebular spectrum of ZTF18alrxas shows an excess around the $\mathrm{H} \alpha$ line, which can be explained through interaction between the SN ejecta and a dense CSM (Section 4.2). Incidentally, all of the well-studied SNe IIb in the literature that show very strong cooling signatures in their early LCs (due to their large radii at the time of explosion) also show signs of CSM interaction in their nebular spectra (SN 1993J, SN 2013df). While not as well studied, this is also likely the case for SN 2011fu (Kumar et al. 2013; MoralesGaroffolo et al. 2015, although the interpretation of this excess is different in Morales-Garoffolo et al. 2015). In contrast, no dense CSM (no significant excess around the $\mathrm{H} \alpha$ region in nebular spectra) is generally seen in $\mathrm{SNe} \mathrm{IIb}$ that are more compact at the time of explosion and lack strong cooling signatures (e.g., SN 2011dh, SN 2008ax; see Jerkstrand et al. 2015, and PTF12os; see Fremling et al. 2016). Thus, as already suggested by Chevalier \& Soderberg (2010), it appears that there are indeed two distinct classes of $\mathrm{SNe} \mathrm{IIb}$; one compact class, with less massive $\mathrm{H}$ envelopes without a dense CSM, and one extended class with more massive $\mathrm{H}$ envelopes and a dense CSM (see also Ben-Ami et al. 2015). Here we have added another member, ZTF18aalrxas, to the extended envelope SN IIb family.

In binary model systems for SE SNe, the mass-loss rate (and the potential for the presence of a dense CSM) is driven by the binary interactions (e.g., Smith 2014). Based on this, Maeda et al. (2015) proposed that the extended and compact classes of $\mathrm{SNe}$ IIb represent two different binary evolution paths. Extended SNe IIb (such as SN 1993J) would be exploding when strong binary interaction and subsequent mass loss is ongoing, while compact SNe IIb (such as SN 2011dh) should have a significant delay between the mass-loss episode where most of the $\mathrm{H}$ envelope is lost and the explosion.

More recently, Yoon et al. (2017) have constructed a large grid of binary models for $\mathrm{SNe} \mathrm{IIb}$, and the results from this work at least partly disagree with the picture suggested by Maeda et al. (2015). Compact SNe IIb are in Yoon et al. (2017) produced from systems in a tight orbit undergoing early Case B mass transfer, which means there is indeed a significant delay between the strong interaction and mass-loss phase and the explosion. However, Yoon et al. (2017) also showed that extended $\mathrm{SNe} \mathrm{IIb}$ could be produced through wider orbits that result in late Case B systems, where there is also a significant delay until the explosion. Some of these models for extended $\mathrm{SNe} \mathrm{IIb}$ also seem to produce strong enough winds to be roughly consistent with the presence of CSM interaction (the high mass-loss rate is a function of the radius and final mass at the time of explosion). However, we note that in the current grid, stars with ZAMS masses in the range suitable for ZTF18aalrxas $\left(M_{\text {ZAMS }}=12-13 M_{\odot}\right)$ will not have strong enough mass loss at the time of explosion to explain the CSM signature; a model with $M_{\text {ZAMS }}=13 M_{\odot}$, at solar metallicity, and a final $\mathrm{H}$ mass of roughly $0.15 M_{\odot}$ (consistent with our nebular spectra), will result in a progenitor with $R \approx 640 R_{\odot}$ and $\dot{M} \sim 4 \times 10^{-6} M_{\odot} \mathrm{yr}^{-1}$. The radius of this model is indeed close to what we derive from the early cooling emission of ZTF18aalrxas, but the wind mass loss is around an order of magnitude too low -when compared to the mass-loss rates derived for SN 2013df and SN 1993J. Suitable mass-loss rates are only possible for progenitors with $M_{\text {ZAMS }}>16 M_{\odot}$ in the grid by Yoon et al. (2017), which is not consistent with our LCs and nebular spectrum of ZTF18aalrxas. It is possible that a large enough parameter space has not yet been explored; and ongoing mass transfer might really be needed to have high enough mass loss in the lower-mass progenitor systems. It is possible to have binary systems that produce SN IIb explosions after multiple episodes of mass transfer during both $\mathrm{He}$ and later burning stages (see e.g., Benvenuto et al. 2013, although this model produces a compact SN IIb).

We propose that nebular spectroscopy can be used to test this issue; if a Case B scenario like in Yoon et al. (2017) dominates the production of extended $\mathrm{SNe} \mathrm{IIb}$, then there should be a relation between the ZAMS mass, the CSM density, and the final envelope mass and radius; and a continuum in the strength of the CSM interaction should be seen. If an episode of strong binary interaction must be ongoing at the time of explosion, a high CSM density should be possible for both low- and highprogenitor ZAMS masses, and the CSM interaction should either be non-existent or strong; there should not be a continuum between compact and extended $\mathrm{SNe}$ IIb in terms of the CSM driven late-time $\mathrm{H} \alpha$ emission.

The ZAMS mass that we find for ZTF18aalrxas is similar to that found for SN 2011dh, which is on the lower end for what has been observed in SE SNe, based on their bolometric LCs (e.g., Taddia et al. 2018), and also their late-time oxygen emission (Jerkstrand et al. 2015). The duration of the first LC peak is somewhat shorter in ZTF18aalrxas ( $\approx 6$ days) compared to SN 2013df ( $\approx 12$ days), and we interpret this difference as a lower extended envelope mass for the progenitor of ZTF18aalrxas at the time of explosion compared to SN 2013df (Section 5.1). We also found that SN 1993J appears to have an extended envelope that is nearly identical in mass and extent to that of ZTF18aalrxas. Furthermore, a similar conclusion was 
also reached based on the late-time $\mathrm{H} \alpha$ emission of these $\mathrm{SNe}$ (Section 4.2). The progenitor of SN 1993J is likely the most massive among the progenitors to these extended $\mathrm{SNe} \mathrm{IIb}$ (based on late-time $\mathrm{O}$ emission; Jerkstrand et al. 2015), but it did not have the strongest cooling signature. SN 2011fu is well matched to a $13 M_{\odot}$ ZAMS mass model (Morales-Garoffolo et al. 2015), and had a cooling signature even stronger than SN 2013df. Thus, while the sample is small, it appears that there is currently no relation between ZAMS mass and the strength or duration of the cooling emission in extended $\mathrm{SNe}$ IIb; the ZAMS masses instead appear to be similar in extended $\mathrm{SNe}$ IIb and compact SNe IIb. This could be argued to be in support of the need for an ongoing mass-loss episode at the time of explosion. However, the current sample of objects is far too small to make any real claims; a systematic study of the nebular spectra of a larger sample of SNe IIb is needed.

In conclusion, it appears clear that ZTF18aalrxas, SN 1993J, and SN 2013df are all very similar; they all show strong longlasting cooling signatures in their early LCs, and over time they develop very similar CSM-powered flat-topped $\mathrm{H} \alpha$ profiles in their spectra-indicative of the presence of dense CSM. They likely originate from binary systems with very similar configurations, that result in strong mass loss during the final centuries before the explosions (either as a result of strong winds, or ongoing interaction). We note that as CSM can already be identified clearly at 177 days, it is also likely affecting earlier observations; the result of a dense CSM is generally shallower P-Cygni profiles (see e.g., Dessart et al. 2017), which is exactly what is seen in SN 1993J, SN 2013df, and ZTF18aalrxas, when compared to SN IIb models without any CSM (Figure 4). This also implies that the CSM could be affecting the bolometric luminosity of these SNe, which would lead to overestimated ${ }^{56} \mathrm{Ni}$ masses when they are modeled without taking this into account. This possibility deserves significant future consideration.

This work is based on observations obtained with the Samuel Oschin Telescope $48 \mathrm{inch}$ and the 60 inch Telescope at the Palomar Observatory as part of the Zwicky Transient Facility project. ZTF is supported by the National Science Foundation under grant No. AST-1440341 and a collaboration including Caltech, IPAC, the Weizmann Institute for Science, the Oskar Klein Center at Stockholm University, the University of Maryland, the University of Washington, Deutsches Elektronen-Synchrotron and Humboldt University, Los Alamos National Laboratories, the TANGO Consortium of Taiwan, the University of Wisconsin at Milwaukee, and Lawrence Berkeley National Laboratories. Operations are conducted by COO, IPAC, and UW.

This work was supported by the GROWTH project funded by the National Science Foundation under PIRE grant No. 1545949. The Oskar Klein Centre is funded by the Swedish Research Council.

Part of this research was carried out at the Jet Propulsion Laboratory, California Institute of Technology, under a contract with the National Aeronautics and Space Administration.

This work is also partially based on observations made with the Nordic Optical Telescope, operated by the Nordic Optical Telescope Scientific Association at the Observatorio del Roque de los Muchachos, La Palma, Spain, of the Instituto de Astrofisica de Canarias. Some of the data presented here were obtained with ALFOSC, which is provided by the Instituto de Astrofisica de
Andalucia (IAA) under a joint agreement with the University of Copenhagen and NOTSA.

Some of the data presented herein were obtained at the W. M. Keck Observatory, which is operated as a scientific partnership among the California Institute of Technology, the University of California, and NASA; the observatory was made possible by the generous financial support of the W. M. Keck Foundation.

This Letter is partly based on observations made with the Italian Telescopio Nazionale Galileo (TNG) operated on the island of La Palma by the Fundación Galileo Galilei of the INAF (Istituto Nazionale di Astrofisica) at the Spanish Observatorio del Roque de los Muchachos of the Instituto de Astrofisica de Canarias. It is partially based on observations obtained with the Apache Point Observatory $3.5 \mathrm{~m}$ telescope, which is owned and operated by the Astrophysical Research Consortium.

The Liverpool Telescope is operated on the island of $\mathrm{La}$ Palma by Liverpool John Moores University in the Spanish Observatorio del Roque de los Muchachos of the Instituto de Astrofisica de Canarias with financial support from the UK Science and Technology Facilities Council.

The SED Machine is based upon work supported by the National Science Foundation under grant No. 1106171.

A.G.-Y. is supported by the EU/FP7 via ERC grant 307260 , "The Quantum Universe" I-Core program by the Israeli Committee for planning and budgeting, by ISF, GIF, and Minerva grants, and by the Kimmel award.

This work is part of the research programme VENI, with project No. 016.192.277, which is (partly) financed by the Netherlands Organisation for Scientific Research (NWO).

Software: JEKYLL (Ergon et al. 2018), emcee package (Foreman-Mackey et al. 2013), SkyPortal (Van der Walt et al. 2019), HEAsoft (v6.25; HEASARC 2014), LPipe reduction pipeline (D. A. Perley et al. 2019, in preparation).

\section{ORCID iDs}

C. Fremling (ib https://orcid.org/0000-0002-4223-103X J. Sollerman (iD https://orcid.org/0000-0003-1546-6615 E. Bellm (iD https://orcid.org/0000-0001-8018-5348

N. Blagorodnova (i) https://orcid.org/0000-0003-0901-1606

S. Frederick (ib https://orcid.org/0000-0001-9676-730X

A. Gal-Yam (i) https://orcid.org/0000-0002-3653-5598

D. A. Goldstein (10 https://orcid.org/0000-0003-3461-8661

M. Kasliwal (1) https://orcid.org/0000-0002-5619-4938

S. R. Kulkarni (iD https://orcid.org/0000-0001-5390-8563

T. Kupfer (iD https://orcid.org/0000-0002-6540-1484

F. J. Masci (i) https://orcid.org/0000-0002-8532-9395

A. A. Miller (i) https://orcid.org/0000-0001-9515-478X

D. A. Perley (i) https://orcid.org/0000-0001-8472-1996

U. D. Rebbapragada (i) https://orcid.org/0000-0002-2560-3495

R. Riddle (i) https://orcid.org/0000-0002-0387-370X

S. Schulze (i) https://orcid.org/0000-0001-6797-1889

L. Tartaglia (i) https://orcid.org/0000-0003-3433-1492

Lin Yan (1) https://orcid.org/0000-0003-1710-9339

\section{References}

Ahn, C. P., Alexandroff, R., Allende Prieto, C., et al. 2014, ApJS, 211, 17 Arcavi, I., Gal-Yam, A., Yaron, O., et al. 2011, ApJL, 742, L18 Bellm, E. C., Kulkarni, S. R., Graham, M. J., et al. 2019, PASP, 131, 018002 Ben-Ami, S., Hachinger, S., Gal-Yam, A., et al. 2015, ApJ, 803, 40 Benvenuto, O. G., Bersten, M. C., \& Nomoto, K. 2013, ApJ, 762, 74 
Bersten, M. C., Folatelli, G., García, F., et al. 2018, Natur, 554, 497 Blagorodnova, N., Neill, J. D., Walters, R., et al. 2018, PASP, 130, 035003 Brown, P. J., Holland, S. T., Immler, S., et al. 2009, AJ, 137, 4517 Brown, T. M., Baliber, N., Bianco, F. B., et al. 2013, PASP, 125, 1031 Cano, Z. 2013, MNRAS, 434, 1098

Cardelli, J. A., Clayton, G. C., \& Mathis, J. S. 1989, ApJ, 345, 245

Cenko, S. B., Fox, D. B., Moon, D.-S., et al. 2006, PASP, 118, 1396 Chambers, K. C., Magnier, E. A., Metcalfe, N., et al. 2016, arXiv:1612.05560 Chevalier, R. A., \& Fransson, C. 1994, ApJ, 420, 268

Chevalier, R. A., \& Soderberg, A. M. 2010, ApJL, 711, L40

Claeys, J. S. W., de Mink, S. E., Pols, O. R., Eldridge, J. J., \& Baes, M. 2011, A\&A, 528, A131

Coughlin, M. W., Dekany, R. G., Duev, D. A., et al. 2019, arXiv:1901.04625 Crockett, R. M., Eldridge, J. J., Smartt, S. J., et al. 2008, MNRAS, 391, L5 Dessart, L., \& Hillier, D. J. 2005, A\&A, 439, 671

Dessart, L., John Hillier, D., \& Audit, E. 2017, A\&A, 605, A83

Drout, M. R., Soderberg, A. M., Gal-Yam, A., et al. 2011, ApJ, 741, 97

Ergon, M., Fransson, C., Jerkstrand, A., et al. 2018, A\&A, 620, A156

Ergon, M., Jerkstrand, A., Sollerman, J., et al. 2015, A\&A, 580, A142

Ergon, M., Sollerman, J., Fraser, M., et al. 2014, A\&A, 562, A17

Fang, Q., \& Maeda, K. 2018, ApJ, 864, 47

Filippenko, A. V. 1997, ARA\&A, 35, 309

Filippenko, A. V., Matheson, T., \& Ho, L. C. 1993, ApJL, 415, L103

Foreman-Mackey, D., Hogg, D. W., Lang, D., \& Goodman, J. 2013, PASP, 125,306

Fox, O. D., Azalee Bostroem, K., Van Dyk, S. D., et al. 2014, ApJ, 790, 17

Fransson, C., Challis, P. M., Chevalier, R. A., et al. 2005, ApJ, 622, 991

Fransson, C., Lundqvist, P., \& Chevalier, R. A. 1996, ApJ, 461, 993

Fremling, C., Sollerman, J., Kasliwal, M. M., et al. 2018, A\&A, 618, A37

Fremling, C., Sollerman, J., Taddia, F., et al. 2016, A\&A, 593, A68

Gal-Yam, A. 2017, in Handbook of Supernovae, ed. A. W. Alsabti \& P. Murdin (Dordrecht: Springer), 195

Gehrels, N., Chincarini, G., Giommi, P., et al. 2004, ApJ, 611, 1005

Graham, M. J., Kulkarni, S. R., Bellm, E. C., et al. 2019, arXiv:1902.01945

Groh, J. H., Meynet, G., Georgy, C., \& Ekström, S. 2013, A\&A, 558, A131

Hamuy, M., Deng, J., Mazzali, P. A., et al. 2009, ApJ, 703, 1612

HEASARC 2014, HEAsoft: Unified Release of FTOOLS and XANADU, Astrophysics Source Code Library, ascl:1408.004

Hinshaw, G., Larson, D., Komatsu, E., et al. 2013, ApJS, 208, 19

Jerkstrand, A., Ergon, M., Smartt, S. J., et al. 2015, A\&A, 573, A12

Kasliwal, M. M., Cannella, C., Bagdasaryan, A., et al. 2019, PASP, 131, 038003
Kumar, B., Pandey, S. B., Sahu, D. K., et al. 2013, MNRAS, 431, 308 Lyman, J. D., Bersier, D., James, P. A., et al. 2016, MNRAS, 457, 328 Maeda, K., Hattori, T., Milisavljevic, D., et al. 2015, ApJ, 807, 35

Masci, F. J., Laher, R. R., Rusholme, B., et al. 2019, PASP, 131, 018003

Matheson, T., Filippenko, A. V., Barth, A. J., et al. 2000a, AJ, 120, 1487

Matheson, T., Filippenko, A. V., Ho, L. C., Barth, A. J., \& Leonard, D. C. 2000b, AJ, 120, 1499

Maund, J. R., \& Smartt, S. J. 2009, Sci, 324, 486

Morales-Garoffolo, A., Elias-Rosa, N., Benetti, S., et al. 2014, MNRAS, 445, 1647

Morales-Garoffolo, A., Elias-Rosa, N., Bersten, M., et al. 2015, MNRAS, 454, 95

Nagy, A. P., \& Vinkó, J. 2016, A\&A, 589, A53

Nakar, E., \& Piro, A. L. 2014, ApJ, 788, 193

Nakar, E., \& Sari, R. 2010, ApJ, 725, 904

Nomoto, K., Suzuki, T., Shigeyama, T., et al. 1993, Natur, 364, 507

Oke, J. B., Cohen, J. G., Carr, M., et al. 1994, SPIE Proc., 2198, 178

Pastorello, A., Kasliwal, M. M., Crockett, R. M., et al. 2008, MNRAS, 389,955

Patat, F., Chugai, N., \& Mazzali, P. A. 1995, A\&A, 299, 715

Piro, A. L. 2015, ApJL, 808, L51

Prentice, S. J., Mazzali, P. A., Pian, E., et al. 2016, MNRAS, 458, 2973

Rabinak, I., \& Waxman, E. 2011, ApJ, 728, 63

Richmond, M. W., Treffers, R. R., Filippenko, A. V., et al. 1994, AJ, 107, 1022

Roming, P. W. A., Kennedy, T. E., Mason, K. O., et al. 2005, SSRv, 120, 95 Rubin, A., \& Gal-Yam, A. 2016, ApJ, 828, 111

Schlafly, E. F., \& Finkbeiner, D. P. 2011, ApJ, 737, 103

Schmidt, B. P., Kirshner, R. P., Eastman, R. G., et al. 1993, Natur, 364, 600

Shivvers, I., Filippenko, A. V., Silverman, J. M., et al. 2019, MNRAS, 482, 1545

Shivvers, I., Mazzali, P., Silverman, J. M., et al. 2013, MNRAS, 436, 3614

Smith, N. 2014, ARA\&A, 52, 487

Szalai, T., Vinkó, J., Nagy, A. P., et al. 2016, MNRAS, 460, 1500

Taddia, F., Sollerman, J., Leloudas, G., et al. 2015, A\&A, 574, A60

Taddia, F., Stritzinger, M. D., Bersten, M., et al. 2018, A\&A, 609, A136

Van der Walt, S. J., Crellin-Quick, A., \& Bloom, J. S. 2019, JOSS, 4, 1247

Van Dyk, S. D., Zheng, W., Fox, O. D., et al. 2014, AJ, 147, 37

Woosley, S. E., \& Heger, A. 2007, PhR, 442, 269

Yaron, O., \& Gal-Yam, A. 2012, PASP, 124, 668

Yoon, S.-C., Dessart, L., \& Clocchiatti, A. 2017, ApJ, 840, 10

Yoon, S.-C., Woosley, S. E., \& Langer, N. 2010, ApJ, 725, 940 